\title{
Impaired Off-Line Consolidation of Motor Memories After Combined Blockade of Cholinergic Receptors During REM Sleep-Rich Sleep
}

\author{
Björn Rasch*,',2, Steffen Gais' and Jan Born*,' \\ 'Department of Neuroendocrinology, University of Lübeck, Lübeck, Germany; '2Department of Molecular Psychology, University of Basel, Basel, \\ Switzerland
}

\begin{abstract}
Rapid eye movement (REM) sleep has been considered important for the consolidation of memories, particularly of procedural skills. REM sleep, in contrast to slow-wave sleep (SWS), is hallmarked by the high, wake-like activity of the neurotransmitter acetylcholine (ACh), which promotes certain synaptic plastic processes underlying the formation of memories. Here, we show in healthy young men that off-line consolidation of a motor skill during a period of late sleep with high amounts of REM sleep depends essentially on high cholinergic activity. After a 3-h sleep period during the early night to satisfy the need for SWS, subjects learned a procedural finger sequence tapping task and a declarative word-pair learning task. After learning, they received either placebo or a combination of the muscarinic receptor antagonist scopolamine $(4 \mu \mathrm{g} / \mathrm{kg}$ bodyweight, intravenously) and the nicotinic receptor antagonist mecamylamine ( $5 \mathrm{mg}$, orally), and then slept for another $3 \mathrm{~h}$, ie, the late nocturnal sleep period, which is dominated by REM sleep. Retrieval was tested the following evening. Combined cholinergic receptor blockade significantly impaired motor skill consolidation, whereas word-pair memory remained unaffected. Additional data show that the impairing effect of cholinergic receptor blockade is specific to sleepdependent consolidation of motor skill and does not occur during a wake-retention interval. Taken together, these results identify high cholinergic activity during late, REM sleep-rich sleep as an essential factor promoting sleep-dependent consolidation of motor skills. Neuropsychopharmacology (2009) 34, I843- 1853; doi:I0.1038/npp.2009.6; published online 4 February 2009
\end{abstract}

Keywords: REM sleep; memory consolidation; acetylcholine; scopolamine; mecamylamine; skill memory

\section{INTRODUCTION}

The cholinergic system modulates brain activity through a network of neural fibers originating in the basal forebrain and tegmental regions and spreading to the entire neocortex, amygdala, and hippocampus (Everitt and Robbins, 1997). Levels of acetylcholine (ACh) in the central nervous system are high during wakefulness, drop to a minimum during slow-wave sleep (SWS), and reach closeto-waking levels again during rapid eye movement (REM) sleep (Pace-Schott and Hobson, 2002; McCarley, 2007). High levels of brain ACh activity are considered a prerequisite for efficient encoding of memories in the

\footnotetext{
* Correspondence: Professor J Born, Department of Neuroendocrinology, University of Lübeck, Ratzeburger Allee 160, Hs 23a, Lübeck D-23538, Germany, Tel: + 49 45I 500 3639, Fax: + 49 45I 5003640 , E-mail: born@kfg.mu-luebeck.de or

Dr. rer. nat. B Rasch, Department of Molecular Psychology, University of Basel, Missionsstrasse 60/62, 4055 Basel, Switzerland,

Tel: +4| 61 26705 9l, Fax: +4| 61 26705 87,

E-mail: bjoern.rasch@unibas.ch

Received II November 2008; revised II December 2008; accepted 19 December 2008
}

wakeful state (Bartus, 2000; Bartus et al, 1982; Rasch et al, 2006). The neuromodulator also plays an important role in the induction of synaptic plasticity during long-term potentiation (Rasmusson, 2000), an experimental paradigm believed to underlie the formation of long-term memories (Cooke and Bliss, 2006).

Sleep benefits the consolidation of newly acquired memories (Born et al, 2006; Walker and Stickgold, 2006). Although consolidation of declarative, hippocampus-dependent memories of events and facts depends particularly on periods of SWS and on low cholinergic activity after learning (Marshall and Born, 2007; Gais and Born, 2004; Rasch et al, 2006), long-term acquisition of skills has been associated repeatedly with REM sleep after training (Born et al, 2006), during which cholinergic tone regains wake-like levels. Numerous animal studies have shown that REM sleep is enhanced after learning of complex procedural tasks, and deprivation of REM sleep, by selective awakenings during certain time windows after learning, impairs subsequent task performance (see Smith, 1995; Peigneux et al, 2001, for a review). Similar findings have been obtained in humans, especially for the retention and consolidation of complex motor skills (Smith et al, 2004; Buchegger and Meier-Koll, 
1988; Buchegger et al, 1991; Karni et al, 1994; Plihal and Born, 1997). Effects of REM sleep deprivation, however, can vary depending on the method used to prevent REM sleep. Thus, pharmacological REM sleep suppression by noradrenergic and serotonergic agonists did not impair skill consolidation, indicating that the mechanisms subserving procedural memory consolidation act partially independent of phenotypic REM sleep features as defined by polysomnography (Rasch et al, 2008).

Although REM sleep is characterized by changes in a number of other neuromodulators, high cholinergic tone might be one of the conditions critical to the consolidating influence of this sleep stage on skills. Support for this notion comes from animal studies showing that suppression of cholinergic neurotransmission, during times after learning that are also vulnerable to REM sleep deprivation (REM sleep windows), consistently impairs memory in different tasks (Smith et al, 1991; Legault et al, 2004, 2006). Conversely, enhancing the cholinergic tone by administration of acetylcholinesterease inhibitors, during post-training sleep, in elderly humans improved consolidation of a procedural skill (Hornung et al, 2007).

In this study, we asked whether the high cholinergic tone associated with REM sleep is critical for procedural memory consolidation. Suppression of cholinergic neurotransmission was achieved by simultaneous administration of the muscarinic receptor antagonist scopolamine and the nicotinic receptor antagonist mecamylamine, during a period of late nocturnal sleep known to be naturally dominated by REM sleep. A combined cholinergic receptor blockade was used, because global alterations in the cholinergic tone accompanying changes in sleep-wake states affect equally both the muscarinic and nicotinic cholinergic receptors. After $3 \mathrm{~h}$ of early, SWS-rich sleep, 11 healthy young men were awakened and trained on a procedural finger sequence tapping task and a declarative word-pair task. After training, they received either placebo or the cholinergic receptor blockers and slept for another $3 \mathrm{~h}$. Retrieval was tested the following evening. We show that the simultaneous cholinergic receptor blockade impairs sleep-dependent procedural memory consolidation. Declarative memory consolidation remained unaffected by the treatment. In supplementary experiments, we show that the impairing effect of cholinergic receptor blockade on skill consolidation is specific to sleep and does not occur during wakefulness.

\section{MATERIALS AND METHODS}

\section{Subjects}

Twelve healthy men (mean age 22.3 years, range 20-26 years) participated in the experiments. Subjects were nonsmoking, native German-speaking, right-handed students. They underwent a routine physical and mental health examination prior to participation, did not take any medication at the time of the experiments, and reported a normal sleep-wake cycle. The participants were instructed to get up at 0700 hours on experimental days, and during these days not to take any naps and not to ingest alcohol or (after 1500 hours) caffeine-containing drinks. To accustom subjects to sleeping under laboratory conditions, all subjects spent an adaptation night in the sleep laboratory, including the placement of electrodes, before the experiments proper. The experiments were approved by the ethics committee of the University of Lübeck. Written informed consent was obtained from all subjects prior to participation.

\section{Design and Procedure}

Each subject participated in two experimental conditions, separated by an interval of at least 2 weeks. The study was conducted according to a double blind crossover design, with one session serving to assess the effects of cholinergic receptor blockade and the other as placebo control. The order of conditions was balanced across subjects. Sessions started at 2100 hours. with the placement of a venous catheter for substance administration and blood collection. After attachment of electrodes for standard polysomnography, participants went to bed at 2230 hours and were allowed to sleep. Three hours after sleep onset, subjects were awakened as soon as sleep stages 1 or 2 occurred. Fifteen minutes after awakening (ca. 0200 hours), they completed two memory tasks, first a declarative verbal paired associate task (word-pairs) and then a procedural finger sequence tapping task. Oral capsules containing either placebo or mecamylamine were administered immediately after learning. Thirty minutes after learning (0330 hours), subjects went back to bed and placebo or scopolamine was infused intravenously. After $3 \mathrm{~h}$ of sleep, subjects were awakened and stayed under observation of the experimenter for another $3.5 \mathrm{~h}$ (until $\sim 1000$ hours). Thereafter, participants could choose to engage in different activities outside the lab. They were not allowed to nap or to take in any caffeine- or alcohol-containing drinks. Adherence to these instructions was confirmed in a postexperimental interview. Retrieval on the two memory tasks was tested $\sim 12 \mathrm{~h}$ after substance administration, starting at 1630 hours with the word-pair learning task followed by the finger sequence tapping task. Before learning, as well as before and after sleep and before retrieval testing, reaction time, mood, feelings of tiredness and calmness/restlessness, and subjective symptoms were measured. Blood for determination of plasma cortisol concentrations was sampled before sleep at 2200 hours, before and after learning ( $\sim 0200$ and 0300 hours), and before retrieval the next day (1600 hours). In addition, blood sampling occurred hourly during early and late sleep. Blood pressure and heart rate were measured at the same time points. A summary of the procedure is given in Figure 1.

\section{Substance Administration}

To block cholinergic transmission, we administered a combination of the muscarinic receptor antagonist scopolamine ( $4 \mu \mathrm{g} / \mathrm{kg}$ bodyweight, intravenously over $20 \mathrm{~min})$ and the nicotinic receptor antagonist mecamylamine $(5 \mathrm{mg}$, orally). Both substances readily cross the blood-brain barrier, exert central nervous effects already at low doses, and have no or negligible affinity to receptors of the other major neurotransmitter systems (Young et al, 2001; Renner et al, 2005). The muscarinic and nicotinic cholinergic receptors are expressed widely in the brain, particularly 


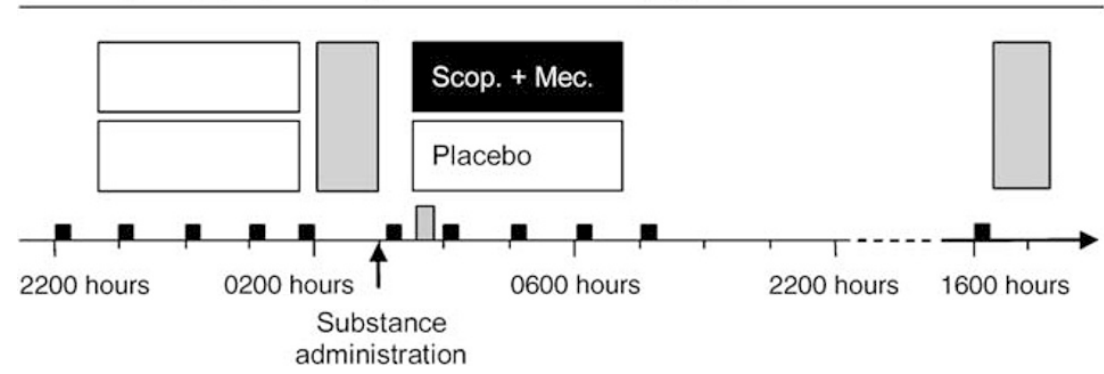

Figure I Experimental procedure and design. We measured the effects of placebo and combined cholinergic receptor blockade, after learning, before REM sleep-rich late nocturnal sleep on memory consolidation (late retention sleep). Combined cholinergic receptor blockade (Scop. + Mec.) was achieved by the joint administration of the muscarinic receptor antagonist scopolamine (gray box) and the nicotinic receptor antagonist mecamylamine (arrow). Before learning, during the early night, subjects had slept for $3 \mathrm{~h}$ to satisfy the need for SWS (early sleep). The experiment was conducted according to a double blind, within-subject, crossover design. The learning phase included training on a procedural memory task (finger sequence tapping) and learning of a declarative memory task (paired associate learning). Retrieval was tested $12 \mathrm{~h}$ after substance administration. Black squares on the time axis indicate times of measuring blood pressure and blood cortisol levels.

in regions considered important for sleep and memory (brain stem, tegmental regions, hippocampus, amygdala, neocortex, etc.) (Court et al, 2000; Eckelman, 2006). To account for the longer time interval of the orally administered drug to reach the brain, mecamylamine was administered immediately after learning (0330 hours), and scopolamine was infused starting $30 \mathrm{~min}$ after the end of the learning phase (ie, at the time of lights off; Figure 1). We chose relatively low doses to keep the side effects of cholinergic receptor blockade at a minimum, and to ensure that substances had largely washed out at the time of retrieval testing. The half-life in plasma is estimated at $4.5 \pm 1.7 \mathrm{~h}$ for scopolamine (Putcha et al, 1989) and $10.1 \pm 2 \mathrm{~h}$ for mecamylamine (Young et al, 2001). In the control condition, subjects received placebo.

\section{Tasks}

The finger sequence tapping task was adopted from earlier studies, indicating very robust sleep-dependent improvements in this task (Walker et al, 2002). It requires the subject to press repeatedly 1 of 25 -element sequences (4-1$3-2-4$ or $4-2-3-1-4)$ with the fingers of the non-dominant hand on a keyboard as fast and as accurately as possible for 30 -s epochs interrupted by 30 -s breaks. The numeric sequence was displayed on the screen at all times to keep working memory demands at a minimum. A key press resulted in a white dot in the center of the screen. Each 30-s trial was scored for speed (number of correctly completed sequences) and error rate (number of errors relative to total number of tapped sequences). After each 30-s trial, feedback was given about the number of correctly completed sequences and error rate. At learning, subjects trained on twelve 30-s trials. The average score for the last three of these trials was used to indicate learning performance. At retrieval, subjects were tested on another three trials. Overnight changes in performance were calculated as absolute differences in speed and error rate between the three trials at retrieval and the last three trials at learning.

As a control for the specificity of effects on procedural memory, a declarative verbal paired associate task was employed requiring the learning of a list of 40 pairs of semantically related words (eg, clock-church). Different wordlists were used on the subject's two experimental sessions. During the learning phase, the word-pairs were presented sequentially on a computer screen, each for $5 \mathrm{~s}$, separated by interstimulus intervals of $100 \mathrm{~ms}$. After presentation of the entire list, performance was tested using a cued-recall procedure, ie, the first word (cue) of each pair was presented and the subject had to name the associated second word (response). The correct response word was then displayed for $2 \mathrm{~s}$, regardless of whether the response was correct or not, to allow re-encoding of the correct wordpair. The cued-recall procedure was repeated until the subject reached a criterion of $60 \%$ correct responses. Retrieval at the end of the experimental session was tested using the same cued-recall procedure as during the learning phase. Absolute differences between word-pairs recalled at retrieval testing and on the criterion trial during learning, served as a dependent variable of overnight retention of declarative memories. Several studies showed that consolidation of word-pairs profits particularly from SWS, whereas sleep periods rich in REM sleep remained ineffective (Ekstrand et al, 1977; Plihal and Born, 1997).

\section{Reaction Times, Mood, Cardiovascular and Neuroendocrine Measures}

Reaction times were assessed as a measure of vigilance by a standardized test that required pressing a button as fast as possible whenever a big red disk appeared on a computer screen (Little et al, 1998). In 40 trials, the subjects fixed their gaze on a cross, displayed for $500-1000 \mathrm{~ms}$ on a white screen. Then, in 35 trials, a red disk appeared, and, in five random no-go trials, the screen remained white.

Mood and feelings of tiredness were assessed using the short form of the German version of the Multidimensional Mood Questionnaire (Steyer et al, 1994). The subjects indicated on a 5-point rating scale how well 12 different adjectives described their current feeling. The adjectives are assigned to one of three different bipolar dimensions, pleasant/unpleasant, alert/tired, and calm/restless, adding up to values between 4 and 20 . At the end of the session, subjects were asked to report any unusual symptoms they had experienced. 
Blood pressure and heart rate were measured with a digital blood pressure meter (Boso-Medicus, Bosch \& Sohn $\mathrm{GmbH}$, Jungingen, Germany). For determination of cortisol concentrations, blood samples were centrifuged immediately, and serum was stored at $-20^{\circ} \mathrm{C}$ until standard assay (Immulite, DPC Biermann, Bad Nauheim, Germany).

\section{EEG Recordings and Analysis of Sleep Parameters}

The EEG was recorded continuously using a SynAmps amplifier (NeuroScan Laboratories, Sterling, VA). EEG signals were filtered between 0.16 and $35 \mathrm{~Hz}$ and sampled at a rate of $250 \mathrm{~Hz}$. Twenty-eight $\mathrm{Ag}-\mathrm{AgCl}$ electrodes were placed according to the 10-20 System, referenced to an electrode attached to the nose. Additionally, horizontal and vertical eye movements (HEOG, VEOG) and the electromyogram (chin) were recorded for standard polysomnography.

Sleep architecture was determined according to standard polysomnographic criteria using EEG recordings from C3 and C4 (Rechtschaffen and Kales, 1968). Scoring was carried out independently by two experienced technicians who were blind to the assigned treatment. In case of disagreement, a third expert was consulted. For the total time in bed, every 30 -s epoch was scored as non-REM sleep stages 1, 2, 3, 4 or REM sleep with SWS defined by the sum of time in sleep stages 3 and 4 . Sleep onset was defined by the first period in stage 1 sleep followed immediately by stage 2 sleep. Sleep onset latency was determined with reference to the time when lights were turned off. REM sleep latency was determined with reference to sleep onset.

Average REM density was calculated by dividing the number of 1-s periods during REM sleep that contained rapid-eye movements by the total number of 30-s REM sleep epochs (Ficca et al, 2004). REMs during REM sleep were detected automatically and were defined as rapid signal changes in the HEOG and VEOG channel $(>0.8 \mathrm{mV} / \mathrm{s})$ after movement artefact rejection and application of a 50 ms moving average.

Spindles (counts and density) in these sleep stages were analyzed because of their well-known relationship with overnight retention of memories (Gais et al, 2002; Nishida and Walker, 2007; Fogel et al, 2007). Discrete spindles are a characteristic feature of sleep stage 2 and also occur in SWS, but are virtually absent during REM sleep. Slow $(<13 \mathrm{~Hz})$ and fast spindles $(>13 \mathrm{~Hz})$ were identified separately at six selected EEG recording sites (F3, F4, C3, C4, P3, P4) during non-REM sleep stages 2,3 , and 4 , based on an algorithm adopted from earlier studies (Schimicek et al, 1994; Gais et al, 2002). In brief, the EEG signal was filtered in the frequency bands of interest $(10-13,13-16 \mathrm{~Hz})$, the root mean square of each $100-\mathrm{ms}$ interval was calculated, and the events were counted for which the root mean square signal exceeded an individual threshold for an interval lasting 0.5$3 \mathrm{~s}$. The detection threshold was defined by the 3-fold average SD across all the six EEG channels analyzed, and across the subject's two experimental nights (active agent and placebo). Spindles were counted separately in each channel. Mean spindle counts were calculated by averaging spindle counts of all the six channels. To calculate spindle density, mean spindle counts were divided by the number of analyzed 30 -s epochs. The two separate spindle bands were chosen based on earlier studies which showed the presence of two kinds of spindles in humans possibly linked to different aspects of cognitive function, ie, slow spindles that prevail over the frontal cortex, and show greater topographical variability than the fast spindles that concentrate over the parietal cortex (Zeitlhofer et al, 1997; Schabus et al, 2007).

\section{Statistical Analysis}

One subject was excluded from the analyses because he slept only for $1.5 \mathrm{~h}$ instead of $3 \mathrm{~h}$ during late sleep in the placebo condition (outlier criterion: $>3$ SDs apart from group mean). Data were analyzed using repeated measures analyses of variance. For the memory tasks, learning performance (at the end of training) and overnight changes (absolute differences between performance at retrieval minus performance at learning) served as dependent variable in separate analyses. Accuracy data from the finger sequence tapping task were log transformed prior to analysis to account for deviations from normal distribution because of positive skewness. For analyses of blood pressure, heart rate, and blood cortisol concentrations, values were averaged to obtain mean values during the early sleep period before learning, during learning, and during retention (late) sleep and retrieval, respectively. A twotailed $P$-value $<0.05$ was considered significant.

\section{Supplementary Experiments}

To examine whether the effects of cholinergic receptor blockade on memory consolidation are sleep specific, we conducted supplementary experiments investigating the effects of combined cholinergic receptor blockade after training on skill consolidation during wakefulness (portions of this data were published elsewhere; Rasch et al, 2006). Twelve age-matched healthy men (mean age 23.2 years, range 21-25 years) carried out the finger sequence tapping task in the morning (0900-1000 hours), and received either placebo or mecamylamine and scopolamine $30 \mathrm{~min}$ after training in a within-subject crossover design. Substance doses were the same as in the main experiment. Retrieval was tested the evening after $9.5 \mathrm{~h}$ of sustained wakefulness.

\section{RESULTS}

\section{Sleep Parameters}

Total sleep time during the retention sleep period, in the late night (after substance administration), was closely comparable between the conditions of combined cholinergic receptor blockade $(180 \pm 9 \mathrm{~min})$ and placebo $(178 \pm 8 \mathrm{~min} ; P>0.8)$. However, the cholinergic receptor blockade suppressed REM sleep (Table 1). In the placebo condition, REM sleep averaged $21.7 \pm 3.2 \%$ of total late sleep time and occurred with a latency of $60 \pm 12 \mathrm{~min}$ after sleep onset. After the cholinergic receptor blockade REM sleep was reduced to only $7.2 \pm 1.6 \%$ of sleep time $(P<0.01)$, and occurred later $(109 \pm 15 \mathrm{~min} ; P<0.04)$. These effects were comparable with that observed in earlier studies, which used serotonergic or noradrenergic substances to suppress REM sleep, but found no impairment of 
Table I Sleep Parameters

\begin{tabular}{|c|c|c|c|c|c|c|}
\hline & \multicolumn{2}{|c|}{ Early sleep } & \multirow[b]{2}{*}{$P$} & \multicolumn{2}{|c|}{ Late retention sleep } & \multirow[b]{2}{*}{$P$} \\
\hline & $\begin{array}{c}\text { Placebo } \\
\text { Mean } \pm \text { SEM }\end{array}$ & $\begin{array}{l}\text { Scop.+Mec. } \\
\text { Mean } \pm \text { SEM }\end{array}$ & & $\begin{array}{c}\text { Placebo } \\
\text { Mean } \pm \text { SEM }\end{array}$ & $\begin{array}{l}\text { Scop.+Mec. } \\
\text { Mean } \pm \text { SEM }\end{array}$ & \\
\hline Wake \% & $6.2 \pm 3.2$ & $7.7 \pm 3.5$ & NS & $3.8 \pm 1.4$ & $2.7 \pm 0.9$ & NS \\
\hline Stage 1\% & $7.5 \pm 0.8$ & $9.7 \pm 1.6$ & NS & $10.0 \pm 1.5$ & $9.9 \pm 1.4$ & NS \\
\hline REM sleep \% & $8.5 \pm 2.0$ & $7.9 \pm 1.6$ & NS & $21.7 \pm 3.2$ & $7.2 \pm 1.6$ & $<0.0$ । \\
\hline Total sleep time (min) & $174 \pm 1 \mid$ & $183 \pm 7$ & NS & $178 \pm 8$ & $180 \pm 9$ & NS \\
\hline Sleep latency (min) & $19 \pm 6$ & $13 \pm 4$ & NS & $26 \pm 5$ & $18 \pm 2$ & NS \\
\hline REM latency (min) & $95 \pm 11$ & $97 \pm 16$ & NS & $60 \pm 12$ & $109 \pm 15$ & $<0.04$ \\
\hline Slow spindle density & $1.13 \pm 0.07$ & $1.07 \pm 0.06$ & NS & $1.07 \pm 0.10$ & $1.13 \pm 0.11$ & NS \\
\hline Fast spindle number & $298 \pm 35$ & $333 \pm 27$ & NS & $288 \pm 32$ & $347 \pm 34$ & NS \\
\hline Fast spindle density & $1.18 \pm 0.14$ & $1.28 \pm 0.04$ & NS & $1.30 \pm 0.14$ & $1.28 \pm 0.13$ & NS \\
\hline
\end{tabular}

NS, not significant; REM, rapid eye movement; Scop.+Mec., scopolamine and mecamylamine; SEM. standard error of mean; SWS, slow wave sleep. Sleep parameters for early sleep (before substance administration) and late retention sleep (after substance administration) for the administration of placebo or a combination of the cholinergic receptor antagonists Scop.+Mec. Time periods of intermittent wakefulness (wake), stages I and 2 sleep, SWS, and REM sleep are indicated as percentages of total sleep time. Total sleep time, sleep latency (with reference to lights off), and REM sleep latency (with reference to sleep onset) are indicated in minutes. REM density is calculated as an average number of I-s periods containing REMs per 30-s epoch of stage REM. Spindle number and density (count per 30-s epochs of stage 2 and SWS) is indicated separately for slow $(<13 \mathrm{~Hz})$ and fast spindles $(>13 \mathrm{~Hz})$. Mean values, SEM, and $P$-values for pair-wise comparisons are indicated. $P>0.05$ is NS.

procedural memory consolidation (Rasch et al, 2008). In addition, density of REMs (average number of eye movements per $30 \mathrm{~s}$ ) in the remaining REM sleep epochs was reduced by the cholinergic receptor blockade to $2.1 \pm 0.5$, compared with $5.3 \pm 0.9$ in the placebo condition $(P<0.01)$. The suppression of REM sleep was compensated by a significant increase in stage 2 sleep percentage in the treatment condition $(66.0 \pm 3.4 \%)$ compared with that in placebo $(54.4 \pm 2.7 ; P<0.04)$. No effect of the cholinergic receptor blockade on slow or fast spindle density was observed (all $P>0.1$ ). The cholinergic receptor blockade did not affect any other sleep stage (all $P>0.1$ ). Sleep architecture during the early sleep period (before substance administration) did not differ between the two experimental conditions (all $P>0.1$, Table 1).

\section{Memory Tasks}

In accordance with our hypothesis, cholinergic blockade after learning impaired procedural memory consolidation during late retention sleep. The number of correctly tapped sequences (speed) in the finger sequence tapping task improved significantly across retention sleep after placebo administration $(+24.0 \pm 4.9 \% ; P<0.001)$, whereas only a marginal improvement occurred with scopolamine and mecamylamine administration after learning (+ $10.9 \pm 5.3 \% ; P<0.07)$. The difference between overnight gains in tapping speed was highly significant $(P<0.01$; Figure 2; see Table 2 for absolute values). The number of

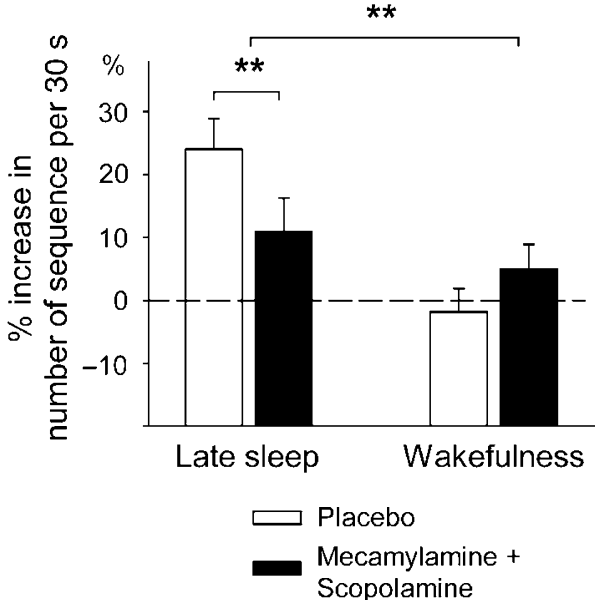

Figure 2 Effects of post-learning cholinergic receptor blockade on procedural memory consolidation during late retention sleep (late sleep, left) and sustained wakefulness (right). Increases in the number of correctly tapped sequences (per 30-s trial) at retrieval testing compared with that in training are indicated as positive gains in speed, with performance at learning set to $100 \%$. Compared with placebo, off-line gains in tapping speed were reduced significantly after cholinergic receptor blockade during REM sleep-rich retention sleep $(* * P<0.0 I)$. No off-line gains were observed after a retention interval filled with wakefulness. Analysis of variance revealed a significant interaction between the type of post-training treatment (placebo vs cholinergic receptor blocker) and the type of retention interval (late retention sleep vs wakefulness; $* * P<0.0$ I). Means \pm SEMs are shown. 
Table 2 Memory Performance

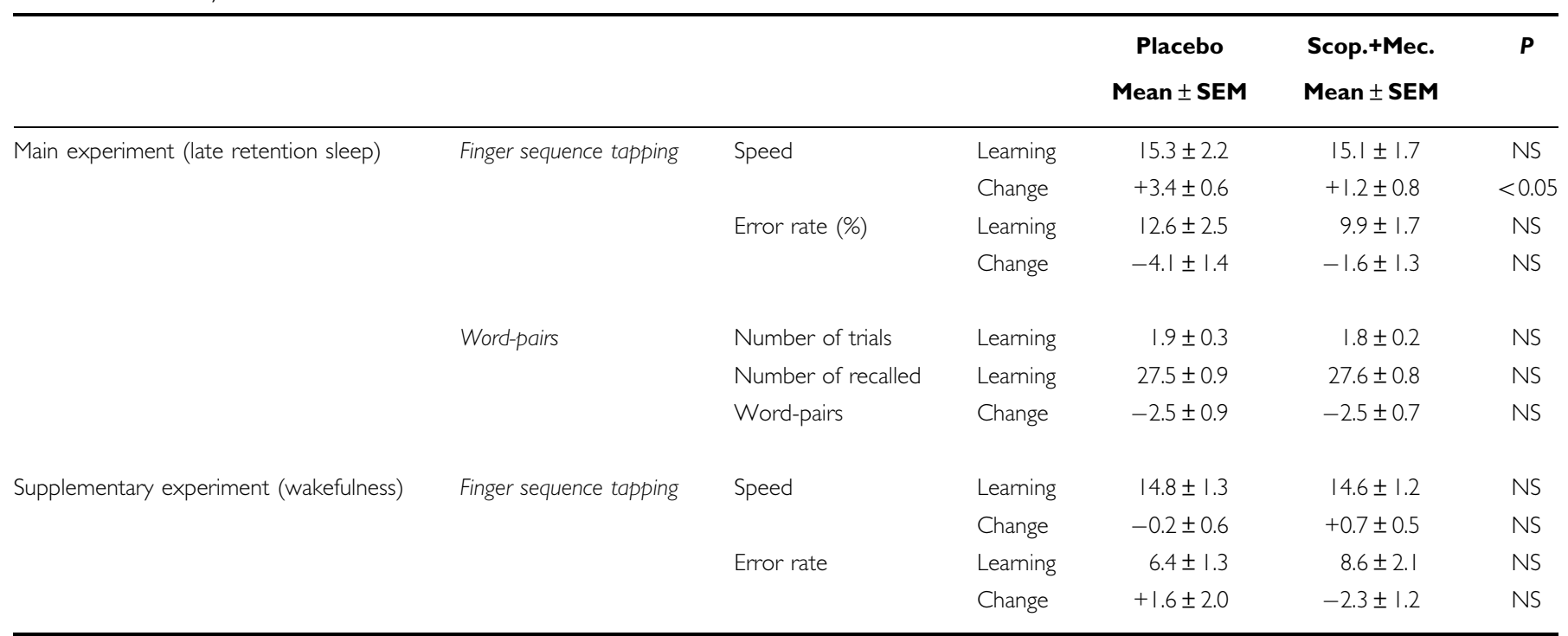

NS, not significant; Scop.+Mec., scopolamine and mecamylamine; SEM, standard error of mean.

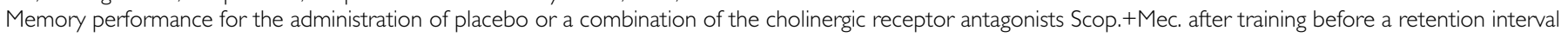

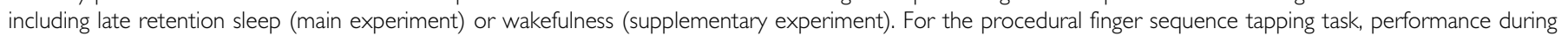
learning is indicated as speed (number of correctly tapped sequences) and error rate (errors per sequence in \%). For the declarative word-pair learning task, performance during learning is indicated as number of trials to the criterion of $60 \%$ and the number of correctly recalled word-pairs in the last training trial. Performance changes are calculated as absolute difference between memory performance during learning and retrieval (retrieval minus learning). Data are mean values, SEM, and $P$-values for pair-wise comparisons. $P>0.05$ is NS.

erroneous sequences relative to the number of all tapped sequence (error rate) was reduced after late retention sleep with placebo $(-4.1 \pm 1.4 \% ; P<0.02)$, whereas error rate did not improve after retention sleep with the cholinergic receptor blockade $(-1.6 \pm 1.3 \% ; P>0.2)$. However, gains in error rate did not differ significantly between the two experimental conditions $(P>0.3)$. Performance during learning before substance administration was comparable for both speed (placebo: $15.3 \pm 2.2$ sequences; cholinergic blockade: $15.1 \pm 1.7$ sequences; $P>0.8$ ) and error rate (placebo: $12.6 \pm 2.5 \%$; cholinergic blockade: $9.9 \pm 1.7 \%$; $P>0.3)$. Although the cholinergic receptor blockade distinctly reduced REM sleep, no significant correlation was observed between the difference in sleep parameters and the improvement differences in finger sequence performance between treatment and placebo nights (all $P>0.1$ ).

In contrast to procedural memory consolidation, retention of declarative memories across late sleep was not affected by the combined cholinergic blockade. When tested the next evening, participants had equally forgotten (with reference to criterion performance at learning) $2.5 \pm 0.9$ word-pairs in the placebo condition and $2.5 \pm 0.7$ word-pairs in the treatment condition $(P>0.9$; Table 2$)$. At learning, neither the number of correctly recalled word-pairs in the last training trial (placebo: $27.5 \pm 0.9$; cholinergic blockade: $27.6 \pm 0.8 ; P>0.9$ ) nor the number of trials to reach the criterion (placebo: $1.9 \pm 0.3$; cholinergic blockade: $1.8 \pm 0.2 ; P>0.7$ ) differed between the two treatment conditions.

\section{Reaction Times, Mood, Cardiovascular and Neuroendocrine Measures}

During learning and retrieval testing, participants did not differ with regard to subjective ratings of mood, wakefulness or calmness between treatment and placebo conditions (all $P>0.1$; Table 3). At the time of retrieval testing, none of the participants reported any anticholinergic symptom (such as dry mouth, etc.). Reaction time was also not affected by substance administration ( $245 \pm 6$ vs $246 \pm 7 \mathrm{~ms}$, for placebo and treatment sessions, respectively; $P>0.8$ ). As expected, the combined cholinergic blockade, after learning, reduced blood pressure during retention sleep (placebo: $111 \pm 3$ / $67 \pm 2 \mathrm{~mm} \mathrm{Hg}$; cholinergic blockade: $103 \pm 3 / 61 \pm 2 \mathrm{~mm} \mathrm{Hg}$, for systolic-diastolic blood pressure, respectively; both $P<0.05)$. At retrieval testing the following evening, systolic blood pressure did not differ between conditions (placebo: $131 \pm 4 \mathrm{~mm} \mathrm{Hg}$; cholinergic blockade: $127 \pm 5 \mathrm{~mm} \mathrm{Hg}$; $P>0.4$ ), whereas diastolic blood pressure was still lower in the cholinergic blockade condition (placebo: $79 \pm 3 \mathrm{~mm} \mathrm{Hg}$; cholinergic blockade: $69 \pm 3 \mathrm{~mm} \mathrm{Hg} ; \quad P<0.05)$. However, this decrease in diastolic blood pressure did not correlate with differences in overnight changes in memory performance (all $r<0.1 ; P>0.7)$, rendering any confounding effects of reduced diastolic blood pressure during retrieval testing highly unlikely. Heart rate was significantly lower during late night sleep after the combined cholinergic receptor blockade (52 \pm 1 b.p.m) compared with that in placebo ( $58 \pm 2$ b.p.m; $P<0.02)$. No significant difference in heart rate occurred before substance administration (early sleep, learning), as well as during retrieval testing (all $P>0.05$ ). Plasma levels of cortisol were comparable between the experimental conditions during learning and retrieval testing, as well as during early and late sleep (all $P>0.1$, Table 3).

\section{Supplementary Experiments}

To examine whether the effects of cholinergic receptor blockade on procedural finger tapping performance at the 
Table 3 Reaction Times, Mood, Blood Pressure, and Cortisol Levels

\begin{tabular}{|c|c|c|c|c|}
\hline & & $\begin{array}{c}\text { Placebo } \\
\text { Mean } \pm \text { SEM }\end{array}$ & $\begin{array}{l}\text { Scop.+Mec. } \\
\text { Mean } \pm \text { SEM }\end{array}$ & $\mathbf{P}$ \\
\hline \multirow[t]{2}{*}{$\mathrm{RT}$ (ms) } & Learning & $248 \pm 7.9$ & $258 \pm 9.2$ & NS \\
\hline & Retrieval & $245 \pm 5.9$ & $246 \pm 7.4$ & NS \\
\hline \multirow[t]{2}{*}{ Mood } & Learning & $14.0 \pm 1.0$ & $13.6 \pm 0.9$ & NS \\
\hline & Retrieval & $13.5 \pm 1.2$ & $15.4 \pm 0.6$ & NS \\
\hline \multirow[t]{2}{*}{ Wakefulness } & Learning & $10.2 \pm 0.9$ & $10.0 \pm 1.0$ & NS \\
\hline & Retrieval & $11.6 \pm 1.2$ & $11.3 \pm 1.1$ & NS \\
\hline \multirow[t]{2}{*}{ Calmness } & Learning & $14.3 \pm 0.9$ & $14.0 \pm 0.9$ & NS \\
\hline & Retrieval & $13.8 \pm 1.2$ & $|5| \pm 0.6$. & NS \\
\hline \multirow{4}{*}{$\begin{array}{l}\text { Systolic blood } \\
\text { pressure }(\mathrm{mm} \mathrm{Hg})\end{array}$} & Early sleep & $108 \pm 2$ & $110 \pm 2$ & NS \\
\hline & Learning & $113 \pm 3$ & $114 \pm 3$ & NS \\
\hline & Late sleep & $111 \pm 3$ & $103 \pm 3$ & $<0.02$ \\
\hline & Retrieval & $|3| \pm 4$ & $127 \pm 3$ & NS \\
\hline \multirow{4}{*}{$\begin{array}{l}\text { Diastolic blood } \\
\text { pressure }(\mathrm{mm} \mathrm{Hg})\end{array}$} & Early sleep & $69 \pm 2$ & $65 \pm 2$ & NS \\
\hline & Learning & $68 \pm 2$ & $67 \pm 2$ & NS \\
\hline & Late sleep & $67 \pm 2$ & $61 \pm 2$ & $<0.04$ \\
\hline & Retrieval & $79 \pm 3$ & $69 \pm 3$ & $<0.02$ \\
\hline \multirow[t]{4}{*}{ Heart rate (b.p.m) } & Early sleep & $62 \pm 2$ & $58 \pm 2$ & NS \\
\hline & Learning & $60 \pm 2$ & $55 \pm 2$ & NS \\
\hline & Late sleep & $58 \pm 2$ & $52 \pm 1$ & $<0.01$ \\
\hline & Retrieval & $70 \pm 4$ & $72 \pm 4$ & NS \\
\hline \multirow[t]{4}{*}{ Cortisol (mmol/l) } & Early sleep & $49 \pm 8$ & $52 \pm 6$ & NS \\
\hline & Learning & $154 \pm 27$ & $182 \pm 32$ & NS \\
\hline & Late sleep & $173 \pm 25$ & $215 \pm 27$ & NS \\
\hline & Retrieval & $227 \pm 27$ & $222 \pm 25$ & NS \\
\hline
\end{tabular}

NS, not significant; RT, reaction time; Scop.+Mec., scopolamine and mecamylamine; SEM, standard error of mean.

RT (as a measure of vigilance), self-reported mood, wakefulness and restlessness, as well as blood pressure and blood cortisol during learning and at retrieval testing for the administration of placebo or a combination of the cholinergic receptor antagonists Scop.+Mec. Blood pressure, heart rate, and cortisol levels were additionally measured hourly during early sleep and during late retention sleep. Mean values, SEM, and $P$-values for pair-wise comparisons are indicated. $P>0.05$ is NS.

time of retrieval testing pertain to off-line consolidation processes specific to sleep, we compared the effects of combined scopolamine-mecamylamine administration and placebo after learning on procedural memory consolidation during wakefulness. In contrast to the results of the main experiment, tapping speed did not improve across the wake-retention interval and did not differ between the placebo $(-1.8 \pm 3.7 \%)$ and treatment condition $(+5.0 \pm 3.9 \%$; $P>0.2$; Figure 2). Changes in error rate were also not affected by the combined cholinergic blockade during the wake-retention interval (placebo: $+1.6 \pm 2.0$; cholinergic blockade: $-2.3 \pm 1.2 ; \quad P>0.1)$. Performance during learning did not differ between the two conditions (speed $14.8 \pm 1.0$ vs $14.6 \pm 1.2$; error rate: $6.4 \pm 1.3 v s$ $8.6 \pm 2.1$, for placebo $v s$ treatment conditions, respectively; both $P>0.4$ ).

Additionally, we tested the specificity of the effect of cholinergic blockade after training on procedural memory consolidation by directly comparing the data from the main and supplementary experiments using a $2 \times 2$ analysis of variance. This analysis confirmed a significant interaction effect between the influence of placebo $v s$ cholinergic blockade and the type of retention interval (late sleep $v s$ wakefulness) on performance changes in the finger sequence tapping task $\left(F_{(1,21)}=8.6 ; P<0.01\right.$; Figure 2$)$. No main effect of cholinergic blockade after training was observed $(P>0.1)$. A similar pattern was observed for changes in error rate, although here the interaction effect reached only a statistical trend $\left(F_{(1,21)}=3.4 ; P<0.08\right)$. These results indicate that the impairing effect of combined cholinergic receptor blockade on consolidation of the finger sequence tapping task is specific to late retention sleep and does not occur during a retention interval filled with wakefulness.

\section{DISCUSSION}

The combined blockade of nicotinergic and muscarinergic ACh receptors during a period of late sleep with natural predominance of REM sleep impaired off-line consolidation of a motor skill. In the placebo condition, as expected, substantial gains in the speed of finger sequence tapping performance developed across the retention interval filled with REM sleep-rich sleep. These gains were abolished when the cholinergic receptors were blocked during this late sleep interval. Declarative memory consolidation was not affected by anticholinergic treatment. Blocking the cholinergic receptors during a wake-retention interval did not affect skill performance at retrieval testing, indicating that the impairing effect of anticholinergic treatment on skill memory is specific to sleep-dependent processes of consolidation. Although the treatment reduced REM sleep, earlier experiments show that pharmacological suppression of phenotypic REM sleep alone (eg, by serotonergic or norepinephrinergic agonists) is not sufficient to impair procedural memory performance (Rasch et al, 2008). On this background, these results identify the high cholinergic tone characterizing REM sleep as a factor that in combination with other unknown mechanisms supports the effective off-line consolidation of motor skills.

Apart from high cholinergic activity, REM sleep is hallmarked by minimum central nervous serotonergic and noradrenergic activity. Surprisingly, although drastically reducing REM sleep, enhancing the availability of norepinephrine or serotonin by administration of reuptake inhibitors in an earlier study using the same memory tasks, did not induce any signs of impaired motor skill consolidation during sleep (Rasch et al, 2008). Thus, in contrast to the high ACh activity, a low aminergic tone or the phenotypic signs of REM sleep are not required for 
successful consolidation of motor skills during sleep. However, cholinergic activity associated with wakefulness is not sufficient for enhancing memory consolidation. In combination, those and the data presented here, suggest that specific components like the enhanced cholinergic tone together with other to-be-defined features of REM sleep are interacting in the off-line development of sleep-dependent gains in skills. On the other hand, the occurrence of phenotypic REM sleep is not necessary.

Candidate mechanisms supporting memory consolidation during REM sleep possibly involve increased protein synthesis and plasticity-related gene expression during this sleep stage. Whereas non-REM sleep is characterized by diminished activity of such genes and a greatly reduced ability to induce synaptic long-term potentiation, REM sleep provides a more adequate milieu of neurotransmitters and modulators for synaptic plastic changes (Ribeiro and Nicolelis, 2004; Bramham and Srebro, 1989). Ribeiro et al. (Ribeiro et al, 1999, 2002) revealed an increased expression of the immediate early genes arc and zif-268 during REM sleep after induction of long-term potentiation during prior wakefulness. Importantly, regulation of plasticity-related early genes depends strongly on the cholinergic receptor activation (von der Kammer et al, 1998, 2001; Teber et al, 2004) and, here, blocking these receptors interfered likely with transcriptional changes required for long-term memory storage. This view is corroborated by recent evidence showing that cholinergic activation potentiates long-term potentiation maintenance in the medial prefrontal cortex (Lopes et al, 2008), a brain area that exhibited particularly strong sleep-dependent changes in activation during the same skill learning task as used here (Walker et al, 2005; Fischer et al, 2005). We suspect that the combined cholinergic receptor blockade during late, REM sleep-rich sleep interfered specifically with processes of synaptic consolidation required for sleep-dependent enhancements of skill performance (Dudai, 2004).

Our results are consistent with the findings in older adults showing that enhancing phasic REM sleep, ie, periods during REM sleep including REMs, improved consolidation of motor memories only, when the enhancement was accomplished by pharmacologically increasing availability of ACh by administration of the acetylcholinesterase inhibitor donezepil (Hornung et al, 2007). In contrast, enhancement of phasic REM sleep by inducing rebound activity (after REM deprivation) had no effect on memory performance, although phasic REM sleep increased to an extent similar to that after the pharmacological manipulation. Further support for the importance of ACh for processes of memory consolidation during REM sleep is provided by animal studies showing that administration of cholinergic receptor antagonists, after training, impairs memory consolidation during time windows that are also vulnerable for REM sleep deprivation (Smith et al, 1991; Legault et al, 2004). Blockade of cholinergic neurotransmission was particularly effective with infusion into the dorsal striatum, a brain structure considered important for learning and performance of skills (Legault et al, 2006; Knowlton et al, 1996; Featherstone and McDonald, 2005). Although these studies did not report any sleep recording, the naturally high cholinergic tone during REM sleep may be required for the induction and/or the maintenance of synaptic changes in striatal regions, underlying the effective consolidation of procedural skills.

High levels of ACh during REM sleep affect both the nicotinic and muscarinic cholinergic receptors, which are both distributed widely in brain areas involved in memory and sleep regulation (Court et al, 2000; Eckelman, 2006). Activation of the nicotinic receptors specifically enhances interest afferent input to cortical and hippocampal networks, whereas muscarinic receptor activation inhibits excitatory feedback synapses in these circuits (see Hasselmo, 2006, for a review). In combination, these mechanisms are considered to enhance signal-to-noise ratio during stimulus processing. During REM sleep, the same mechanisms might act in concert with a cholinergic facilitation of synaptic plasticity to promote skill consolidation (Power, 2004). In this study, we blocked simultaneously the muscarinic and nicotinic receptors to mimic global suppression of cholinergic activity as it occurs in specific brain states like SWS. To what extent the two receptor types contributed differentially to the effects of receptor blocking cannot be answered. In an earlier study (Rasch et al, 2006), that showed enhanced retention but impaired encoding of declarative memories after combined administration of scopolamine and mecamylamine, separate administration of either one of the receptor blockers (at the same dose used for combined administration) remained completely ineffective. Such findings support the view that changes in memory processing after the combined blockade of nicotinic and muscarinic receptors reflect the result of globally diminished cholinergic transmission that cannot be achieved by separate blockade of one of the two receptor types. Moreover, the separation of muscarinic and nicotinic contributions is hampered by interactions between the receptors. For example, selectively blocking the muscarinic receptors by scopolamine, through blocking M2 presynaptic autoreceptors, induces a pronounced increase in ACh release, which, in turn, can lead to an overactivation of post-synaptic nicotinic receptors (Maviel and Durkin, 2003; Durkin et al, 1992). Thus, modeling the effects of a generally lowered cholinergic neurotransmission requires that both the cholinergic receptor types are blocked simultaneously, thereby avoiding increased nicotinic activation.

The effect of combined cholinergic receptor blockade impaired procedural memory consolidation only when administered before a retention period of sleep with dominant REM sleep, but not when given during a retention period of sustained wakefulness. On the one hand, this finding excludes any residual substance effects on motor performance at the time of retrieval testing. On the other hand, this finding underscores that the anticholinergic effect is specific for consolidation processes occurring during sleep, in an interaction with other REM sleep-related consolidation mechanisms.

Procedural memory consolidation during sleep has also been related to aspects of non-REM sleep, in particular sleep spindles (Walker et al, 2002; Robertson et al, 2004; Nishida and Walker, 2007; Fogel et al, 2007; Rasch et al, 2008). In this study, we observed an impairment of procedural memory consolidation by the combined cholinergic receptor blockade in spite of a significantly enhanced duration of stage 2 non-REM sleep. Spindle density was not affected by the treatment (see also Hirshkowitz et al, 1982). At a first 
glance, this finding speaks against an involvement of stage 2 sleep in skill consolidation. However, any beneficial effect of this sleep stage may have been masked by the strongly impairing influence of the cholinergic receptor blockade per se. Moreover, we cannot exclude that blocking cholinergic neurotransmission impaired skill consolidation processes, additionally by affecting mechanisms associated with nonREM sleep. Thus, although high cholinergic activity suppresses sleep spindles (Puolivali et al, 1998; Jäkälä et al, 1997), it is still possible that some minimum cholinergic activity is required for spindle activity effectively supporting skill consolidation.

Although, here, we did not test the effects of combined cholinergic blockade during early, SWS-rich sleep, it seems very unlikely that the blocking cholinergic receptors during early sleep would exert any effect on procedural memory consolidation, because the cholinergic tone is already minimal during SWS. In fact, in comparison with posttraining periods of wakefulness, SWS does not substantially benefit the consolidation enhancement of procedural skills (Plihal and Born, 1997, 1999; Mednick et al, 2003). In addition, pharmacologically enhancing the cholinergic tone during early SWS-rich sleep is ineffective for procedural memory (Gais and Born, 2004), whereas enhancing the cholinergic tone during late sleep enhances procedural memory performance (Hornung et al, 2007).

In contrast to procedural memories, the consolidation of declarative word-pairs was not affected by the combined cholinergic blockade after learning during REM sleep-rich sleep. This is in line with earlier studies showing no benefit of declarative memory consolidation from late sleep (Ekstrand et al, 1977; Plihal and Born, 1997, 1999). However, we have shown recently that the combined cholinergic blockade after learning improves retention of word-pairs when applied during a wake-retention interval (Rasch et al, 2006), whereas increasing availability of ACh during early, SWS-rich sleep disrupts the beneficial effect of SWS on declarative memory consolidation (Gais and Born, 2004). Although these two studies suggest a general improving effect on declarative memory consolidation of low cholinergic tone after learning (Hasselmo, 1999), this apparently does not hold for the conditions during late REM sleep-rich sleep. Beyond this, these findings indicate that consolidation during sleep depends on separate mechanisms for different forms of memory, ie, procedural and declarative memories, with REM sleep not providing the appropriate neuromodulatory environment for effective declarative memory consolidation. For example, compared with SWS or wakefulness, serotonin and norepinehrine are less available during REM sleep (Pace-Schott and Hobson, 2002), and moreover, cortisol levels are higher during late REM sleep than early SWS-rich sleep (Born and Fehm, 1998). Increasing cortisol levels during sleep blocks the improving effect of sleep on declarative memory consolidation (Plihal and Born, 1999; Wagner and Born, 2008).

In conclusion, although earlier studies showed that the complex processes that produce the signs of phenotypic REM sleep are not prerequisites for effective sleepdependent consolidation of motor skills (Rasch et al, 2008), specific processes and mechanisms active during natural REM sleep may still result in consolidation of motor skills. This study reveals enhanced cholinergic neuro- transmission as one such factor, which in combination with other so far unknown mechanisms during late, REM sleep-rich sleep promotes the consolidation of procedural skills.

\section{ACKNOWLEDGEMENTS}

We thank J Lingscheidt, C Otten, and A Otterbein for technical assistance. This study was supported by a grant from the Deutsche Forschungsgemeinschaft SFB 654 'Plasticity and Sleep'.

\section{DISCLOSURE/CONFLICT OF INTEREST}

The authors declare that, except for income received from each respective primary employer, no financial support or compensation has been received from any individual or corporate entity over the past 3 years for research or professional service, and there are no personal financial holdings that could be perceived as constituting a potential conflict of interest.

\section{REFERENCES}

Bartus RT (2000). On neurodegenerative diseases, models, and treatment strategies: lessons learned and lessons forgotten a generation following the cholinergic hypothesis. Exp Neurol 163: 495-529.

Bartus RT, Dean RL, Beer B, Lippa AS (1982). The cholinergic hypothesis of geriatric memory dysfunction. Science 217: 408-414.

Born J, Fehm HL (1998). Hypothalamus-pituitary-adrenal activity during human sleep: a coordinating role for the limbic hippocampal system. Exp Clin Endocrinol Diabetes 106: 153-163.

Born J, Rasch B, Gais S (2006). Sleep to remember. Neuroscientist 12: $410-424$.

Bramham CR, Srebro B (1989). Synaptic plasticity in the hippocampus is modulated by behavioral state. Brain Res 493: 74-86.

Buchegger J, Fritsch R, Meier-Koll A, Riehle H (1991). Does trampolining and anaerobic physical fitness affect sleep? Percept Mot Skills 73: 243-252.

Buchegger J, Meier-Koll A (1988). Motor learning and ultradian sleep cycle: an electroencephalographic study of trampoliners. Percept Mot Skills 67: 635-645.

Cooke SF, Bliss TV (2006). Plasticity in the human central nervous system. Brain 129: 1659-1673.

Court J, Martin-Ruiz C, Graham A, Perry E (2000). Nicotinic receptors in human brain: topography and pathology. J Chem Neuroanat 20: 281-298.

Dudai Y (2004). The neurobiology of consolidations, or, how stable is the engram? Annu Rev Psychol 55: 51-86.

Durkin TP, Messier C, de BP, Westerink BH (1992). Raised glucose levels enhance scopolamine-induced acetylcholine overflow from the hippocampus: an in vivo microdialysis study in the rat. Behav Brain Res 49: 181-188.

Eckelman WC (2006). Imaging of muscarinic receptors in the central nervous system. Curr Pharm Des 12: 3901-3913.

Ekstrand BR, Barrett TR, West JN, Meier WG (1977). The effect of sleep on human long-term memory. In: Drucker-Colin RR, McGaugh JL (eds). Neurobiology of Sleep and Memory. Academic Press: New York. pp 419-438.

Everitt BJ, Robbins TW (1997). Central cholinergic systems and cognition. Annu Rev Psychol 48: 649-684.

Featherstone RE, McDonald RJ (2005). Lesions of the dorsolateral striatum impair the acquisition of a simplified stimulus- 
response dependent conditional discrimination task. Neuroscience 136: 387-395.

Ficca G, Scavelli S, Fagioli I, Gori S, Murri L, Salzarulo P (2004). Rapid eye movement activity before spontaneous awakening in elderly subjects. J Sleep Res 13: 49-53.

Fischer S, Nitschke MF, Melchert UH, Erdmann C, Born J (2005). Motor memory consolidation in sleep shapes more effective neuronal representations. J Neurosci 25: 11248-11255.

Fogel SM, Smith CT, Cote KA (2007). Dissociable learningdependent changes in REM and non-REM sleep in declarative and procedural memory systems. Behav Brain Res 180: 48-61.

Gais S, Born J (2004). Low acetylcholine during slow-wave sleep is critical for declarative memory consolidation. Proc Natl Acad Sci USA 101: 2140-2144.

Gais S, Mölle M, Helms K, Born J (2002). Learning-dependent increases in sleep spindle density. J Neurosci 22: 6830-6834.

Hasselmo ME (1999). Neuromodulation: acetylcholine and memory consolidation. Trends Cogn Sci 3: 351-359.

Hasselmo ME (2006). The role of acetylcholine in learning and memory. Curr Opin Neurobiol 16: 710-715.

Hirshkowitz M, Thornby JI, Karacan I (1982). Sleep spindles: pharmacological effects in humans. Sleep 5: 85-94.

Hornung OP, Regen F, nker-Hopfe H, Schredl M, Heuser I (2007). The relationship between REM sleep and memory consolidation in old age and effects of cholinergic medication. Biol Psychiatry 61: 750-757.

Jäkälä P, Puolivali J, Bjorklund M, Koivisto E, Riekkinen Jr P (1997). Activation of acetylcholine receptors and 5-HT2 receptors have additive effects in the suppression of neocortical high-voltage spindles in aged rats. Psychopharmacology (Berl) 132: $270-280$.

Karni A, Tanne D, Rubenstein BS, Askenasy JJ, Sagi D (1994). Dependence on REM sleep of overnight improvement of a perceptual skill. Science 265: 679-682.

Knowlton BJ, Mangels JA, Squire LR (1996). A neostriatal habit learning system in humans. Science 273: 1399-1402.

Legault G, Smith CT, Beninger RJ (2004). Scopolamine during the paradoxical sleep window impairs radial arm maze learning in rats. Pharmacol Biochem Behav 79: 715-721.

Legault G, Smith CT, Beninger RJ (2006). Post-training intrastriatal scopolamine or flupenthixol impairs radial maze learning in rats. Behav Brain Res 170: 148-155.

Little JT, Johnson DN, Minichiello M, Weingartner H, Sunderland $\mathrm{T}$ (1998). Combined nicotinic and muscarinic blockade in elderly normal volunteers: cognitive, behavioral, and physiologic responses. Neuropsychopharmacology 19: 60-69.

Lopes AC, Romcy-Pereira RN, Escorsim SR, Galvis-Alonso OY, nselmo-Franci JA, Pereira LJ (2008). Muscarinic acetylcholine neurotransmission enhances the late-phase of long-term potentiation in the hippocampal-prefrontal cortex pathway of rats in vivo: a possible involvement of monoaminergic systems. Neuroscience 153: 1309-1319.

Marshall L, Born J (2007). The contribution of sleep to hippocampus-dependent memory consolidation. Trends Cogn Sci 11: 442-450.

Maviel T, Durkin TP (2003). Role of central cholinergic receptor sub-types in spatial working memory: a five-arm maze task in mice provides evidence for a functional role of nicotinic receptors in mediating trace access processes. Neuroscience 120: $1049-1059$.

McCarley RW (2007). Neurobiology of REM and NREM sleep. Sleep Med 8: 302-330.

Mednick S, Nakayama K, Stickgold R (2003). Sleep-dependent learning: a nap is as good as a night. Nat Neurosci 6: 697-698.

Nishida M, Walker MP (2007). Daytime naps, motor memory consolidation and regionally specific sleep spindles. PLOS ONE 2: e341.
Pace-Schott EF, Hobson JA (2002). The neurobiology of sleep: genetics, cellular physiology and subcortical networks. Nat Rev Neurosci 3: 591-605.

Peigneux P, Laureys S, Delbeuck X, Maquet P (2001). Sleeping brain, learning brain. The role of sleep for memory systems. Neuroreport 12: A111-A124.

Plihal W, Born J (1997). Effects of early and late nocturnal sleep on declarative and procedural memory. J Cogn Neurosci 9: 534-547.

Plihal W, Born J (1999). Memory consolidation in human sleep depends on inhibition of glucocorticoid release. Neuroreport 10: 2741-2747.

Power AE (2004). Slow-wave sleep, acetylcholine, and memory consolidation. Proc Natl Acad Sci USA 101: 1795-1796.

Puolivali J, Jakala P, Koivisto E, Riekkinen Jr P (1998). Oxotremorine suppresses thalamocortical oscillations via thalamic muscarinic acetylcholine receptors. Psychopharmacology (Berl) 140: 285-292.

Putcha L, Cintron NM, Tsui J, Vanderploeg JM, Kramer WG (1989). Pharmacokinetics and oral bioavailability of scopolamine in normal subjects. Pharm Res 6: 481-485.

Rasch B, Pommer J, Diekelmann S, Born J (2008). Pharmacological REM sleep suppression paradoxically improves rather than impairs skill memory. Nat Neurosci. doi: advance online publication, 5 Oct 2008 (doi:10.1038/nn2206).

Rasch BH, Born J, Gais S (2006). Combined blockade of cholinergic receptors shifts the brain from stimulus encoding to memory consolidation. J Cogn Neurosci 18: 793-802.

Rasmusson DD (2000). The role of acetylcholine in cortical synaptic plasticity. Behav Brain Res 115: 205-218.

Rechtschaffen A, Kales A (1968). A Manual of Standardized Terminology, Techniques and Scoring System for Sleep Stages of Human Subjects. Brain Inf. Service, University of California: Los Angeles.

Renner UD, Oertel R, Kirch W (2005). Pharmacokinetics and pharmacodynamics in clinical use of scopolamine. Ther Drug Monit 27: 655-665.

Ribeiro S, Goyal V, Mello CV, Pavlides C (1999). Brain gene expression during REM sleep depends on prior waking experience. Learn Mem 6: 500-508.

Ribeiro S, Mello CV, Velho T, Gardner TJ, Jarvis ED, Pavlides C (2002). Induction of hippocampal long-term potentiation during waking leads to increased extrahippocampal zif-268 expression during ensuing rapid-eye-movement sleep. J Neurosci 22: 10914-10923.

Ribeiro S, Nicolelis MA (2004). Reverberation, storage, and postsynaptic propagation of memories during sleep. Learn Mem 11: 686-696.

Robertson EM, Pascual-Leone A, Press DZ (2004). Awareness modifies the skill-learning benefits of sleep. Curr Biol 14: 208-212.

Schabus M, ng-Vu TT, Albouy G, Balteau E, Boly M, Carrier J et al (2007). Hemodynamic cerebral correlates of sleep spindles during human non-rapid eye movement sleep. Proc Natl Acad Sci USA 104: 13164-13169.

Schimicek P, Zeitlhofer J, Anderer P, Saletu B (1994). Automatic sleep-spindle detection procedure: aspects of reliability and validity. Clin Electroencephalogr 25: 26-29.

Smith C (1995). Sleep states and memory processes. Behav Brain Res 69: 137-145.

Smith C, Tenn C, Annett R (1991). Some biochemical and behavioural aspects of the paradoxical sleep window. Can J Psychol 45: 115-124.

Smith CT, Nixon MR, Nader RS (2004). Posttraining increases in REM sleep intensity implicate REM sleep in memory processing and provide a biological marker of learning potential. Learn Mem 11: 714-719.

Steyer R, Schwenkmezger P, Notz P, Eid M (1994). Theoretical analysis of a multidimensional mood questionnaire (MDBF). Diagnostica 40: 320-328. 
Teber I, Kohling R, Speckmann EJ, Barnekow A, Kremerskothen J (2004). Muscarinic acetylcholine receptor stimulation induces expression of the activity-regulated cytoskeleton-associated gene (ARC). Brain Res Mol Brain Res 121: 131-136.

von der Kammer H, Demiralay C, Andresen B, Albrecht C, Mayhaus M, Nitsch RM (2001). Regulation of gene expression by muscarinic acetylcholine receptors. Biochem Soc Symp 67: 131-140. von der Kammer H, Mayhaus M, Albrecht C, Enderich J, Wegner M, Nitsch RM (1998). Muscarinic acetylcholine receptors activate expression of the EGR gene family of transcription factors. J Biol Chem 273: 14538-14544.

Wagner U, Born J (2008). Memory consolidation during sleep: interactive effects of sleep stages and HPA regulation. Stress 11: 28-41.
Walker MP, Brakefield T, Morgan A, Hobson JA, Stickgold R (2002). Practice with sleep makes perfect: sleep-dependent motor skill learning. Neuron 35: 205-211.

Walker MP, Stickgold R (2006). Sleep, memory, and plasticity. Annu Rev Psychol 57: 139-166.

Walker MP, Stickgold R, Alsop D, Gaab N, Schlaug G (2005). Sleepdependent motor memory plasticity in the human brain. Neuroscience 133: 911-917.

Young JM, Shytle RD, Sanberg PR, George TP (2001). Mecamylamine: new therapeutic uses and toxicity/risk profile. Clin Ther 23: $532-565$.

Zeitlhofer J, Gruber G, Anderer P, Asenbaum S, Schimicek P, Saletu B (1997). Topographic distribution of sleep spindles in young healthy subjects. J Sleep Res 6: 149-155. 